\title{
Effect of Kangaroo Mother Care on Maternal Anxiety and Depression States at the Neonatal ICU: A Prospective Cohort Study
}

Nicole Rosalie Mallonga-Matilac, MD, Shary May Manuta-Baton, MD and Maria Esterlita T. Villanueva-Uy, MD, MSPH

Philippine General Hospital, University of the Philippines Manila

\begin{abstract}
Objectives. To determine the effect of kangaroo mother care (KMC) on anxiety and depression of mothers of lowbirth-weight neonates during the immediate newborn period.
\end{abstract}

Method. Eligible participants were mothers of low-birth-weight infants (birth weight $\leq 2500$ grams) admitted at a tertiary hospital's neonatal intensive care unit. Mothers were instructed on providing KMC daily to their infants during the study period (first seven days of life).

Main Outcome Measure(s). The primary study outcome was the effect of KMC in improving maternal anxiety and depression scores in the immediate postpartum period using the locally validated Hospital Anxiety and Depression Scale-Pilipino (HADS/HADS-P).

Results. A total of 171 mothers were enrolled in the study. Only 79 mothers provided KMC, and the rest (92) did not provide KMC. The anxiety and depression scores improved significantly from day 1 to 7 postpartum in both groups $(p<0.05)$. Frequency of mothers categorized as having severe anxiety significantly decreased over time whether they provided KMC or not (KMC: $40.5 \%, 13.9 \%, 7.6 \%$ at Day 1 and 7 postpartum and day of discharge; No KMC: $35.9 \%$ and $27.2 \%$ at Day 1 and Day 7 postpartum). There was a significant reduction in the percentage of mothers categorized in the depressed group from Day 1 to Day 7 postpartum, among those who rendered KMC compared with those who did not (KMC: $7.6 \%, 2.5 \%, 0 \%$ at Day 1 and 7 postpartum and the day of discharge vs. No KMC: $7.6 \%$ and $10.9 \%$ at Day 1 and 7 postpartum). There were no significant differences in the anxiety and depression scores at any period between mothers who rendered $\mathrm{KMC}>6$ hours and $\mathrm{KMC} \leq 6$ hours/day.

Conclusion. Anxiety and depression scores significantly decreased over time in both mothers who rendered and did not render KMC to their infants. However, there was a significant reduction in the percentage of mothers categorized as having severe depression over time among those who rendered KMC compared to those who did not. Other factors aside from KMC may affect the maternal anxiety and depression states, such as instability of the infant.

Key Words: KMC, Maternal Anxiety and Depression, HADS-P

\section{INTRODUCTION}

The immediate newborn period is a joyous time to welcome a new member of the family. The anticipation of the last nine months concludes once the baby is delivered. However, the bliss is short-lived when a family is faced with the reality and difficulty of preterm birth or a neonate with low birthweight. The mother, who herself is in postpartum pain, is tasked to take care of another individual, and the

Corresponding author: Nicole Rosalie Mallonga-Matilac, MD Philippine General Hospital

University of the Philippines Manila

Taft Avenue, Ermita, Manila 1000, Philippines

Email: nicolematilac@yahoo.com.ph father is expected to attend to the needs of the weakened mother and his helpless infant. This preterm, low birth weight infant, or both who need intensive care, renders the family financially and emotionally incapacitated, unprepared for the 
situation at hand. Preterm birth is the number one cause of neonatal mortality and, as such, a cause of significant fear and apprehension in the mother and the family. ${ }^{1}$

In a descriptive study done by Lasiuk on the parental traumas associated with preterm birth, none expected their babies to be born preterm. When it occurred, they were caught unprepared for the series of events that would ensue in the delivery room and the neonatal intensive care unit (NICU). In the early days after their baby's birth, the normalcy of parents' everyday life faded into the background, and their only concern was their babies' precarious survival. ${ }^{2}$

A recent systematic review revealed that post-traumatic symptomatology was not uncommon in parents or primary caregivers of premature infants. ${ }^{3}$ One study in that review found that $67 \%$ of mothers with preterm infants (vs. $6 \%$ of a control group consisting of mothers of full-term infants) exhibited post-traumatic stress disorder symptoms. Similarly, Holditch-Davis found that all mothers in their study had at least one post-traumatic symptom, 12 had two symptoms, and 16 had three symptoms post-birth. ${ }^{4}$ Kersting's group reported that mothers of very premature infants showed significantly higher rates of traumatic symptoms at 1-3 days, 14 days, 6 months, and 14 months post-birth than mothers of healthy term infants. ${ }^{5}$ Furthermore, Wereszczak et al. found that caregivers reported vivid memories of their infant's appearance, behavior, pain, procedures, and illness severity even three years after preterm birth. ${ }^{6}$

More recently, an Alberta study employed a prospective cohort to explore the number and severity of acute stress disorder symptoms in parents of preterm infants at 7-10 days and a month post-preterm birth. The authors found that $25 \%$ of respondents ( $28 \%$ of mothers and $17 \%$ of fathers) met diagnostic criteria for acute stress disorder (ASD) at both measurement times. There were also significant depression scores among $43 \%$ of the mothers at 7-10 days and in 35\% of mothers at one month, and $33 \%$ and $17 \%$ of the fathers, respectively. Maternal depression has implications for the mother's psychological well-being and the infant's cognitive and emotional development. ${ }^{7}$ Prior research demonstrates that the infants of depressed mothers are at greater risk for developmental disturbances, which may compound the effects of prematurity.

According to Lasiuk, the trauma the parents experienced was not related to infant characteristics (e.g., gestational age, birth weight, Apgar scores, or length of stay in the NICU), but rather to prolonged uncertainty, lack of agency, disruptions in meaning systems, and alterations in parental role expectations. ${ }^{2}$ It also elaborated on the importance of breastfeeding, kangaroo mother care, and family-centered practices, which are meaningful to the parents to reconstruct their role as caregivers. These activities afford parents a sense of agency, thereby moderating their helplessness.

Kangaroo mother care (KMC) is a technique done in low-birth-weight newborns, especially in preterm infants wherein the infant is held, skin-to-skin, with an adult. ${ }^{1}$
Kangaroo mother care, named for the similarity to how certain marsupials carry their young, was initially developed to care for preterm infants in areas where incubators are either unavailable or unreliable. Much has been said on the positive outcome of $\mathrm{KMC}$ on that of the preterm infant. In studies of the physiological effects of $\mathrm{KMC}$, the results for most variables were within clinically acceptable ranges or the same as those for premature infants under other forms of care. ${ }^{8}$ Body temperature and weight gain are significantly increased, and a meta-analysis showed that the kangaroo position increases the uptake and duration of breastfeeding - investigations of the behavioral effects of KMC show rapid quiescence. The psychosocial impact of $\mathrm{KMC}$ includes reduced stress, enhancement of mother-infant bonding, and positive impact on the family and the infant's cognitive development.

A study by Norouzi involved randomization of 90 Iranian women after a repeat Cesarean section into three groups: $\mathrm{KMC}$, music during $\mathrm{KMC}$, and a control group (no $\mathrm{KMC}$ ) to measure the impact of the said interventions on maternal anxiety state. The study showed a reduction in the severity of the maternal anxiety scores among those provided KMC ( \pm music) compared with the controls. ${ }^{9}$

A prospective study done among 177 low-income mothers with their preterm infants determined the effect of $\mathrm{KMC}$ on maternal depression using the Portuguese version of the Postpartum Depression Screening Scale (PDSS). ${ }^{10}$ Results showed a decrease in the incidence of mothers with depression from the time of NICU admission to KMC discharge $(37.3 \%$ vs. $16.9 \%$; $<$ 0.0001). None developed postpartum depression during the hospital stay. It was then concluded that KMC might lessen maternal depression.

In a nonequivalent control group pretest-posttest of 22 mothers in the $\mathrm{KMC}$ group and 21 in the control group, Lee showed that KMC was effective in mothers' anxiety relief but not in maternal role confidence and maternalinfant attachment of mothers. ${ }^{11}$ However, in the study of Johnson, KMC also improved maternal confidence in caring for premature infants with reported feelings of "being needed" and "feeling comfortable," leading to the increased understanding of the multifaceted advantages of $\mathrm{KMC}$ on maternal attachment behaviors. ${ }^{12}$ In a transcendental (metaphysical) phenomenology study done by Roller of ten women who provided $\mathrm{KMC}$ to their preterm newborns, KMC facilitated bonding and enhanced maternal-infant acquaintance, even in the NICU environment. ${ }^{13}$ Mothers believed that KMC calmed them and their newborns.

Despite the uncertainty of the newborn's outcome, kangaroo care allows the mother to fulfill the duties of caring for her infant by allowing her to interact and bond with her baby. The practice is a simple intervention that helps mothers and caregivers to adjust to their predicament. KMC can potentially alleviate levels of depression and anxiety. Previous studies from other countries showed a positive impact of $\mathrm{KMC}$ in reducing caregiver stress, anxiety, and depression. ${ }^{14}$ 
A local study among medically ill patients admitted in a tertiary hospital shows a prevalence of $26.9 \%$ for depression and $14.3 \%$ for anxiety among the medically sick. ${ }^{15}$ Still, the percentage of patients seeking intervention is only around 1-3\%. However, no studies in the Philippines involved mothers of preterm and low birth weight infants in a tertiary hospital involving mothers from high-risk social and medical backgrounds.

Two screening tools most commonly used to detect Post-Partum Depression are the Edinburgh Post-Partum Depression Scale (EDPS) and the PDSS. The EPDS has been established as a screening tool for detecting women during the perinatal period at risk for depression. ${ }^{16}$ The questionnaire consists of 10 items with a $59-100 \%$ sensitivity and $49-100 \%$ specificity. The PDSS is a 35 item self-report instrument with a sensitivity of $91-94 \%$ and specificity of $72-98 \% .{ }^{17}$ Both tests perform well in detecting anxiety and depression. The shortcomings of such screening instruments are the multitude of the different cut-offs required for women from different cultures, antenatal, during, and post-natal periods and perinatal anxiety.

The Hospital and Anxiety Depression Scale-Pilipino (HADS-P) is a 14-item self-report questionnaire used in this study to detect depression and anxiety among mothers of preterm and low birth weight infants. ${ }^{15}$ It has been validated in the same local tertiary hospital as this study among the medical and surgically ill. It was concluded as a reliable, valid, and practical screening tool with an optimal cut-off score of 11 , possessing a sensitivity of $75 \%$, specificity of $70 \%$, and PPV of $75 \%$. The test is reliable for use in in-patient and out-patient settings.

\section{Research Objective}

To determine the effect of KMC on the level of anxiety and depression of mothers of low-birth-weight neonates during the immediate newborn period.

\section{METHODOLOGY}

\section{Study Design}

This study was a prospective cohort study conducted at a NICU of a local tertiary hospital for four months.

\section{Study Population}

Eligible participants included mothers of all low birth weight infants admitted at the NICU of a local tertiary hospital.

\section{Study Protocol}

After the birth of a low-birth-weight neonate (birth weight of $\leq 2500$ grams), the mother's informed consent was obtained from the investigator or the research assistant. The mothers were instructed on the benefits and the conduct of providing KMC positions to their infants. The mothers were asked to provide KMC to their infants in the first seven days postpartum. The participants were asked to answer the HADS-P at Day 1 and 7 postpartum and at hospital discharge.

\section{Statistical Analysis and Sample Size Estimation}

A minimum of 146 participants was calculated based on a level of significance of $5 \%$, with a power of $80 \%$. The values used for sample size computation were obtained from the reference article by de Alencar et al. ${ }^{10}$ The study recruitment continued until at least 73 mothers were in two groups: those who rendered KMC for the first seven days and those who did not.

The questionnaire used to determine anxiety or depression in the caregiver was the HADS-P. The HADS-P validation study included Obstetrics and Gynecology patients (18.2\%) from the same institution as this study. The HADS-P has been validated in a study by de Guzman ${ }^{15}$ in 2009 of 710 medically ill in-patient Filipinos. The study results showed that the optimal cut-off score for the HADS/ HADS-P was 11 with a sensitivity of $75 \%$, specificity of $70 \%$, and PPV of $75 \%$. The HADS/HADS-P scoring system was as follows: $0-7=$ normal, $8-10=$ borderline abnormal or borderline case, and 11-21 = abnormal or potential case of anxiety/depression. Borderline abnormal scores meant that the patient could be a probable case of anxiety or depression. An abnormal score indicated that the patient was a case of anxiety or depression. However, such a classification should be confirmed with an assessment done by a clinical psychiatrist, which was not included in this study.

\section{Ethical Considerations}

The protocol was submitted for ethical review and approved by the review board. Informed consent was obtained after the mother delivered a preterm or low birth weight infant. In case of overt signs of anxiety and depression, the patient would be referred to the medical staff of the Department of Psychiatry.

\section{Plan of Analysis and Results}

Descriptive statistics were used to summarize the clinical characteristics of the patients. Frequency and proportion were used for nominal variables, median and IQR for ordinal variables, and mean and $\mathrm{SD}$ for interval/ratio variables. Independent Sample T-test, Mann-Whitney U test, and Fisher's Exact/Chi-square test were used to determine the difference of mean, median, and frequency between groups, respectively. All valid data were included in the analysis. Missing variables were neither replaced nor estimated. The null hypothesis was rejected at $0.05 \alpha$-level of significance. STATA 12.0 was used for data analysis.

\section{RESULTS}

A total of 189 mothers enrolled in the study, and 171 were included in the data analysis. Eighteen mothers were 
Table 1. Baseline Characteristics of the Enrolled Mothers

\begin{tabular}{|c|c|c|c|c|}
\hline \multirow{2}{*}{ Profile of Mothers } & \multicolumn{2}{|c|}{ KMC } & \multirow{2}{*}{$\begin{array}{c}\text { No KMC } \\
(\mathrm{n}=92)\end{array}$} & \multirow{2}{*}{ p-value } \\
\hline & $\leq 6$ hours/day $(n=55)$ & $>6$ hours/day $(n=24)$ & & \\
\hline Age (years), Mean $\pm S D$ & $28.5 \pm 6.6$ & $27.4 \pm 6.1$ & $29.4 \pm 7.9$ & 0.4630 \\
\hline \multicolumn{5}{|l|}{ KMC Provider, n, \% } \\
\hline Mother Only & $50(90.9)$ & $18(75.0)$ & $78(84.8)$ & 0.1039 \\
\hline Mother and Father & $4(7.3)$ & $6(25.0)$ & $12(13.0)$ & \\
\hline \multicolumn{5}{|l|}{ Others } \\
\hline Mother/Aunt & $0(0.0)$ & $0(0.0)$ & $1(1.1)$ & \\
\hline Mother/Grandmother & $1(1.8)$ & $0(0.0)$ & $0(0.0)$ & \\
\hline Mother/Father/Grandmother & $0(0.0)$ & $0(0.0)$ & $1(1.1)$ & \\
\hline \multicolumn{5}{|l|}{ Residence, $n, \%$} \\
\hline Within Manila & $24(43.6)$ & $14(58.3)$ & $63(68.5)$ & 0.0123 \\
\hline Outside Manila & $31(56.4)$ & $10(41.7)$ & $29(31.5)$ & \\
\hline \multicolumn{5}{|l|}{ Registered, $n, \%$} \\
\hline Yes & $21(38.2)$ & $14(58.3)$ & 49 (53.3) & 0.1301 \\
\hline No & $34(61.8)$ & $10(41.7)$ & $43(46.7)$ & \\
\hline \multicolumn{5}{|l|}{ Educational Attainment, n, \% } \\
\hline College & $9(16.4)$ & $8(33.3)$ & $15(16.3)$ & 0.1406 \\
\hline Vocational & $4(7.3)$ & $0(0.0)$ & $3(3.3)$ & \\
\hline High School & $37(67.3)$ & $16(66.7)$ & $60(65.2)$ & \\
\hline Elementary & $5(9.1)$ & $0(0.0)$ & $14(15.2)$ & \\
\hline \multicolumn{5}{|l|}{ Gravidity, n, \% } \\
\hline G1 & $21(38.2)$ & $9(37.5)$ & $34(37.0)$ & 0.6162 \\
\hline G2 & $12(21.8)$ & $7(29.2)$ & $21(22.8)$ & \\
\hline G3 & $15(27.3)$ & $5(20.8)$ & $16(17.4)$ & \\
\hline G4 and above & $7(12.7)$ & $3(12.5)$ & $21(22.8)$ & \\
\hline \multicolumn{5}{|l|}{ Maternal Risk Factors } \\
\hline With & $22(40.0)$ & 8 (33.3) & $29(31.5)$ & 0.5736 \\
\hline Without & $33(60.0)$ & $16(66.7)$ & $63(68.5)$ & \\
\hline Hypertension & $10(18.2)$ & $1(4.2)$ & $9(9.8)$ & 0.1434 \\
\hline Gestational Diabetes Mellitus & $6(10.9)$ & $3(12.5)$ & $5(5.4)$ & 0.3564 \\
\hline Preeclampsia & $9(16.4)$ & $3(12.5)$ & $19(20.7)$ & 0.5405 \\
\hline Urinary Tract Infection & $6(10.9)$ & $2(8.3)$ & $3(2.2)$ & 0.0787 \\
\hline
\end{tabular}

excluded; 9 dropped out and 9 died before the $7^{\text {th }}$ day of life (9.5\%). Out of the 171 remaining mothers, 55 provided $\mathrm{KMC} \leq 6$ hours/days $(32.2 \%)$ and 24 provided $\mathrm{KMC}>6$ hours (14.0\%). Ninety-two mothers did not provide KMC to their infants during the first seven days postpartum (53.8\%). (Figure 1)

Baseline characteristics were similar among those who rendered $\mathrm{KMC}<6$ hours, $\geq 6$ hours, and no KMC except for a higher percentage $(68 \%)$ of a mother who did not render KMC residing in Metro Manila. Gravidity, educational attainment, and the presence of co-morbidities did not differ among the groups. (Table 1)

There were also no significant differences in the baseline characteristics among the three groups. Most of the neonates (75.4\%) were admitted to the critical care area (NICU 3), and $24.6 \%$ were admitted to the intermediate care area (NICU 2). Most mothers of newborns who did not provide KMC during the first seven days postpartum (80.4\%) had

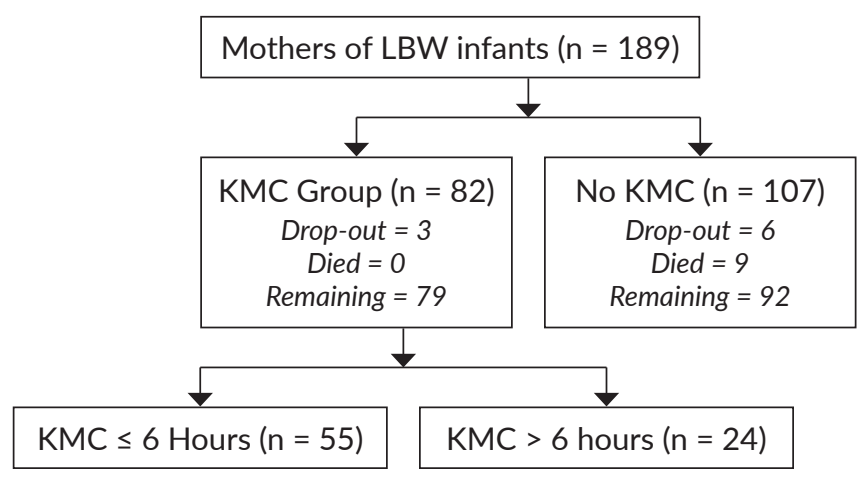

Figure 1. Patient Recruitment and Classification.

infants on the mechanical ventilator. There were 25 newborns (14.6\%) who were unstable during the $1^{\text {st }} 7$ days of life, hence were not eligible for KMC at that time. Still, 10 mothers of stable intubated infants (5.8\%) provided KMC. (Table 2) 
Table 2. Baseline Characteristics of the of Neonates enrolled in the study

\begin{tabular}{|c|c|c|c|c|}
\hline \multirow{2}{*}{ Profile of Neonates } & \multicolumn{2}{|c|}{ KMC } & \multirow{2}{*}{$\begin{array}{c}\text { No KMC } \\
(\mathrm{n}=92)\end{array}$} & \multirow{2}{*}{ p-value } \\
\hline & $\leq 6$ hours/day $(n=55)$ & $>6$ hours/day $(n=24)$ & & \\
\hline Birth weight (grams), Mean \pm SD & $1852.7 \pm 338.9$ & $1777.7 \pm 461.7$ & $1773 \pm 478.2$ & 0.5470 \\
\hline \multicolumn{5}{|l|}{ Gender, $n, \%$} \\
\hline Male & $24(43.6)$ & $11(45.8)$ & $53(57.6)$ & 0.2182 \\
\hline Female & $31(56.4)$ & $13(54.2)$ & $39(42.4)$ & \\
\hline \multicolumn{5}{|l|}{ Size for Gestational Age } \\
\hline Appropriate for Gestational Age & $47(85.5)$ & $22(91.7)$ & 79 (85.9) & 0.7287 \\
\hline Small for Gestational Age & $8(14.5)$ & $2(8.3)$ & $13(14.1)$ & \\
\hline \multicolumn{5}{|l|}{ NICU Level, $n, \%$} \\
\hline 2 (Intermediate Care) & $17(30.9)$ & $7(29.2)$ & $18(19.6)$ & 0.2579 \\
\hline 3 (Critical Care) & $38(69.1)$ & $17(70.8)$ & $74(80.4)$ & \\
\hline \multicolumn{5}{|l|}{ Oxygen Support, $n, \%$} \\
\hline Room Air & $17(30.9)$ & $5(22.7)$ & $19(20.7)$ & 0.3554 \\
\hline Nasal Cannula & $5(9.1)$ & $2(9.1)$ & $11(12.0)$ & 0.9403 \\
\hline Hood & $0(0.0)$ & $1(4.5)$ & $0(0.0)$ & 0.1404 \\
\hline Nasal Continuous Positive Airway Pressure (NCPAP) & $28(50.9)$ & $9(40.9)$ & $37(40.2)$ & 0.3723 \\
\hline Nasal Intermittent Positive Pressure Ventilation (NIPPV) & $0(0.0)$ & $0(0.0)$ & $4(4.3)$ & 0.2747 \\
\hline Intubated & $5(9.1)$ & $5(22.7)$ & $21(22.8)$ & 0.0847 \\
\hline \multicolumn{5}{|l|}{ Diagnosis, $n, \%$} \\
\hline Transient Tachypnea of the Newborn & $14(25.5)$ & $2(8.3)$ & $20(21.7)$ & 0.2678 \\
\hline Neonatal pneumonia & $10(18.2)$ & $4(16.7)$ & $10(10.9)$ & \\
\hline Respiratory distress syndrome & $10(18.2)$ & 9 (37.5) & $19(20.7)$ & \\
\hline Others & $21(38.2)$ & $9(37.5)$ & $43(46.7)$ & \\
\hline
\end{tabular}

Forty-three patients (46.7\%) had diagnoses such as persistent pulmonary hypertension of the newborn, necrotizing enterocolitis, sepsis, neonatal depression, symptomatic hypoglycemia, and various surgical conditions $(\mathrm{CDH}$, jejunal atresia, duodenal atresia) with the mothers of these patients not able to provide KMC during the first seven days because of instability of the newborn.

Both anxiety scores for mothers who provided $\mathrm{KMC}$ and those who did not significantly decrease over time. Similarly, the percentage of mothers classified as having abnormal anxiety severity significantly reduced over time. There was no significant difference between the anxiety scores and the classification between those who rendered KMC and those who did not at any period. (Table 3 )

Some mothers provided KMC > 6 hours/day and others $\leq 6$ hours/day. Anxiety scores and classifications over time significantly improved in both groups. Also, there was no difference in scores and classifications between the two groups at any one time. (Table 4)

There was no significant difference between mean depression scores and classifications between mothers providing $\mathrm{KMC}$ and those who did not at any period. However, there was a considerable reduction in the frequency of mothers classified as having abnormally severe depression among mothers who rendered KMC compared with those who did not (p-value: KMC, 0.0015 vs. No KMC, 0.2574). (Table 5)

Comparing mothers who provided KMC more than 6 hours and those $\leq 6$ hours, there was no significant difference in the mean depression scores at the three-time periods. Both groups showed a substantial reduction in the mean depression scores over the periods analyzed. (Table 6)

\section{DISCUSSION}

All mothers were instructed on enrolment to provide $\mathrm{KMC}$ to their infants in the first seven days of life. Surprisingly, the mothers (68\%) who resided in the metropolis turned out to be the group who did not provide KMC subsequently. It might seem that the mothers who live in Metro Manila could not sustain the daily commute to the hospital due to possible lack of funds, prolonged recovery, or absence of a support person. Furthermore, mothers from far-flung areas were prioritized to be decked to the ten KMC beds allotted by the hospital. Still, the hospital provided lunch or dinner for mothers who would come to provide KMC to their infants to assist them in their daily needs.

Another possible reason the mothers were unable to provide $\mathrm{KMC}$ in the first seven days of life was the instability of their infants. Most neonates (75.4\%) were admitted to the Level III NICU and $24.6 \%$ at the Level II NICU. Most (80.4\%) of the mothers of newborns who did not provide $\mathrm{KMC}$ during the first seven days postpartum had infants on the mechanical ventilator. Of the patients hooked to the ventilator, $25(14.6 \%)$ were unstable, so KMC was not provided. Infants who were not rendered KMC had diagnoses of persistent pulmonary hypertension 
Table 3. Comparison of the Mean Anxiety scores between those who rendered $\mathrm{KMC}$ and those who did not as well as between two time periods

\begin{tabular}{|c|c|c|c|}
\hline Anxiety Score, Mean \pm SD & $\begin{array}{c}\text { KMC } \\
(n=79)\end{array}$ & $\begin{array}{l}\text { No KMC } \\
(n=92)\end{array}$ & p-value \\
\hline 1 Day Post-Partum & $9.4 \pm 3.8$ & $9.4 \pm 3.7$ & 0.4517 \\
\hline 7 Days Post-Partum & $7.0 \pm 3.6$ & $7.9 \pm 4.1$ & 0.0719 \\
\hline Day of Discharge & $4.5 \pm 4.0$ & & \\
\hline$p$-value & 0.0001 & 0.0005 & \\
\hline Classification of Anxiety Severity & $\begin{array}{c}\text { KMC } \\
(n=79)\end{array}$ & $\begin{array}{c}\text { No KMC } \\
(\mathrm{n}=92)\end{array}$ & p-value \\
\hline \multicolumn{4}{|l|}{ Day 1} \\
\hline Abnormal & $32(40.5)$ & $44(35.9)$ & 0.4670 \\
\hline Borderline Abnormal & $19(24.1)$ & $30(32.6)$ & \\
\hline Non-case & $28(35.4)$ & $29(31.5)$ & \\
\hline \multicolumn{4}{|l|}{ Day 7} \\
\hline Abnormal & $11(13.9)$ & $25(27.2)$ & 0.0996 \\
\hline Borderline Abnormal & $26(32.9)$ & $23(25.0)$ & \\
\hline Non-case & 41 (51.9) & $44(47.8)$ & \\
\hline \multicolumn{4}{|l|}{ Day of Discharge } \\
\hline Abnormal & $6(7.6)$ & & \\
\hline Borderline abnormal & $15(19.0)$ & & \\
\hline Non-case & $58(73.4)$ & & \\
\hline$p$-value & 0.0001 & 0.0133 & \\
\hline
\end{tabular}

$p$ value for mean changes from Day 1,7 and discharge

HADS scoring system: 0-7 = normal, 8-10 = Borderline abnormal

(borderline case), 11-21 = abnormal (case)

Table 5. Comparison of the Mean Depression scores between those who rendered KMC and those who did not as well as between two time periods

\begin{tabular}{|c|c|c|c|}
\hline Depression Score, Mean \pm SD & $\begin{array}{c}\text { KMC } \\
(n=79)\end{array}$ & $\begin{array}{l}\text { No KMC } \\
(n=92)\end{array}$ & p-value \\
\hline 1 Day Post-Partum & $5.7 \pm 3.3$ & $5.9 \pm 3.4$ & 0.8106 \\
\hline 7 Days Post-Partum & $5.2 \pm 2.9$ & $5.7 \pm 3.4$ & 0.2435 \\
\hline Day of Discharge & $3.9 \pm 2.8$ & & \\
\hline$p$-value & 0.001 & 0.001 & \\
\hline Depression Score Classification & $\begin{array}{c}\text { KMC } \\
(n=79)\end{array}$ & $\begin{array}{c}\text { No KMC } \\
(n=92)\end{array}$ & p-value \\
\hline \multicolumn{4}{|l|}{ Day 1} \\
\hline Abnormal & $6(7.6)$ & $7(7.6)$ & 0.7555 \\
\hline Borderline Abnormal & $21(26.6)$ & $20(21.7)$ & \\
\hline Non-case & $52(65.8)$ & $65(70.7)$ & \\
\hline \multicolumn{4}{|l|}{ Day 7} \\
\hline Abnormal & $2(2.5)$ & $10(10.9)$ & 0.0765 \\
\hline Borderline Abnormal & 15 (19.0) & $12(13.0)$ & \\
\hline Non-case & $61(77.2)$ & $70(76.1)$ & \\
\hline \multicolumn{4}{|l|}{ Day of Discharge } \\
\hline Abnormal & $0(0.0)$ & & \\
\hline Borderline abnormal & $10(12.7)$ & & \\
\hline Non-case & 69 (87.3) & & \\
\hline $\mathrm{p}$-value & 0.0015 & 0.2574 & \\
\hline
\end{tabular}

HADS scoring system: 0-7 = normal, 8-10 = Borderline abnormal (borderline case), 11-21 = abnormal (case)
Table 4. Comparison of Mean Anxiety scores between mothers who provided KMC > 6 hours/day and those $\leq 6$ hours/day to their infants

\begin{tabular}{|c|c|c|c|}
\hline Anxiety Score, Mean \pm SD & $\begin{array}{l}K M C \leq 6 \\
(n=55)\end{array}$ & $\begin{array}{c}\mathrm{KMC}(>6) \\
(\mathrm{n}=24)\end{array}$ & p-value \\
\hline 1 Day Post-Partum & $9.2 \pm 3.7$ & $9.8 \pm 4.1$ & 0.5405 \\
\hline 7 Days Post-Partum & $6.3 \pm 3.6$ & $8.5 \pm 3.3$ & 0.0166 \\
\hline Day of Discharge & $4.5 \pm 4.1$ & $4.5 \pm 4.0$ & 0.9300 \\
\hline$p$-value & 0.001 & 0.001 & \\
\hline Classification of Anxiety Severity & $\begin{array}{l}K M C \leq 6 \\
(n=55)\end{array}$ & $\begin{array}{c}\mathrm{KMC}(>6) \\
(\mathrm{n}=24)\end{array}$ & p-value \\
\hline \multicolumn{4}{|l|}{ Day 1} \\
\hline Abnormal & $23(41.8)$ & $9(37.5)$ & 0.7806 \\
\hline Borderline Abnormal & $12(21.8)$ & $7(29.2)$ & \\
\hline Non-case & $20(36.4)$ & $8(33.3)$ & \\
\hline \multicolumn{4}{|l|}{ Day 7} \\
\hline Abnormal & $6(10.9)$ & $5(20.8)$ & 0.2469 \\
\hline Borderline Abnormal & $17(30.9)$ & 9 (37.5) & \\
\hline Non-case & $32(58.2)$ & 9 (37.5) & \\
\hline \multicolumn{4}{|l|}{ Day of Discharge } \\
\hline Abnormal & $3(5.5)$ & $3(12.5)$ & 0.3930 \\
\hline Borderline abnormal & $12(21.8)$ & $3(12.5)$ & \\
\hline Non-case & $40(72.7)$ & $18(75.0)$ & \\
\hline $\mathrm{p}$-value & 0.0001 & 0.0147 & \\
\hline
\end{tabular}

$p$ value for mean changes from Day 1, 7 and discharge

HADS scoring system: 0-7 = normal, 8-10 = Borderline abnormal (borderline case), 11-21 = abnormal (case)

Table 6. Comparison of the Mean Depression Scores between mothers who rendered $\mathrm{KMC}>6$ hours and those $\leq 6$ hours

\begin{tabular}{lccc}
$\begin{array}{c}\text { Depression Score, } \\
\text { Mean } \pm \text { SD }\end{array}$ & $\begin{array}{c}\text { KMC } \leq \mathbf{6} \text { hours } \\
(\mathbf{n}=\mathbf{5 5})\end{array}$ & $\begin{array}{c}\text { KMC }>\mathbf{6} \text { hours } \\
(\mathbf{n}=\mathbf{2 4 )}\end{array}$ & p-value \\
\hline 1 Day Post-Partum & $5.7 \pm 3.5$ & $5.8 \pm 2.9$ & 0.8627 \\
\hline 7 Days Post-Partum & $4.9 \pm 3.0$ & $5.9 \pm 2.7$ & 0.1487 \\
\hline Day of Discharge & $3.9 \pm 2.8$ & $3.7 \pm 2.9$ & 0.7542 \\
\hline p-value & 0.001 & 0.001 & \\
\hline
\end{tabular}

HADS scoring system: 0-7 = normal, 8-10 = Borderline abnormal (borderline case), 11-21 = abnormal (case)

of the newborn, necrotizing enterocolitis, sepsis, neonatal depression, symptomatic hypoglycemia, and abdominal surgical conditions. Still, once the infants have been stabilized, whether on respiratory support or not, KMC was offered even after the study period (first seven days).

Anxiety and depression scores improved over time whether the mothers provided $\mathrm{KMC}$ or not to their infant. The mothers enrolled in the study were usually well and discharged early. In the study by De Guzman, those discharged early compared to those who remained admitted in the hospital for more than eight days were less likely to experience anxiety and depression. ${ }^{15}$ The other possible reasons for decreased anxiety among mothers, whether they rendered or did not render $\mathrm{KMC}$, could be early acceptance 
of the infant's condition, trust in the physician, and confidence that things would turn out for the best despite being admitted at the NICU. In this study, some mothers disclosed that they encountered worse problems in the past, which they could overcome. Hence, other factors could also help decrease anxiety in the mother, which were unique for every subject, such as past experiences, level of education, absence of a co-existing medical problem, early discharge from the hospital, and good family support, as was cited in local studies that enabled patients to cope with anxiety and depression. ${ }^{15}$

Over time, there was a significant reduction in the percentage of mothers classified as having considerable depression among the KMC group compared with mothers who could not render KMC. This may be due to the higher number of sicker infants among mothers who could not provide $\mathrm{KMC}$. In these cases, the non-provision of $\mathrm{KMC}$ was not the mother's choice but rather due to the instability of their infants. This may be the reason for the non-improvement in depression classification among these mothers. Furthermore, the KMC study of de Alencar showed that improving depressive symptoms may take time and that familiarity with the newborn and breastfeeding would help lower the risk of depression. ${ }^{10}$ On the day of discharge, the KMC mothers had better scores than their scores at Day 7 postpartum. Mothers who could not render KMC during the first seven days of life were no longer tested at discharge.

The effect of the average duration of KMC on the maternal anxiety and depression scores was also analyzed. There was no significant difference in the scores between the two groups at any period. The study by Lee did not investigate the duration of $\mathrm{KMC}$ but rather the frequency of $\mathrm{KMC}$, three times daily in four days, which showed improvement in anxiety over time. ${ }^{11}$ It is possible that the frequency of $\mathrm{KMC}$ also reduced maternal anxiety apart from duration. As stated in the study by Roller, KMC that facilitated bonding, an acquaintance of mother and infant even in the stressful setting of the NICU calmed both mothers and newborns. ${ }^{13}$

\section{CONCLUSION}

Both mothers' anxiety and depression scores who rendered $\mathrm{KMC}$ and those who did not improve significantly over time. However, mothers who rendered $\mathrm{KMC}$ were less likely to be classified as depressed on day seven postpartum and the day of discharge. Other factors like the instability of the preterm infants may play a role in the depression and anxiety of mothers.

\section{Recommendations}

Longer observation of the psychopathological symptoms of mothers of preterm and LBW infants who rendered $\mathrm{KMC}$ is recommended to determine the lasting effect of $\mathrm{KMC}$ done in the first seven days of life.

\section{Statement of Authorship}

All authors participated in the data collection and analysis and approved the final version submitted.

\section{Author Disclosure}

All authors declared no conflicts of interest.

\section{Funding Source}

This study received a research grant from the Kangaroo Mother Care Foundation Philippines, Inc.

\section{REFERENCES}

1. Howson C, Kinney M, Lawn M. Born Too Soon: The Global Action Report on Preterm Birth. March of Dimes, PMNCH, Save the Children, World Health Organization.Geneva; 2012 [cited 2015 Feb]: Available from [http://whqlibdoc.who.int/publications/ 2012/9789241503433_ eng.pdf].

2. Lasiuk G, Comeau T, Newburn-Cook C. Unexpected: an interpretive description of parental traumata associated with preterm birth. BMC Pregnancy Childbirth. 2013;13 Suppl 1: S13. doi: 10.1186/1471-239313-S1-S13. Epub 2013 Jan 31.

3. Pierrhumbert B, Nicole A, Muller-Nix C, Forcada-Guex M, Ansermet F:Parental post-traumatic reactions after premature birth: implications for sleeping and eating problems in the infant. Arch Dis Child Fetal Neonatal Ed. 2005, 88:400-404.

4. Holditch-Davis D, Bartlett TR, Blickman AL, Shandor Miles M: Posttraumatic stress symptoms in mothers of premature infants. JOGNN 2003, 32:161-171.

5. Kersting A, Dorsch M, Wesselmann U, Ludorff K, Witthaut J, Ohrmann P, et al.: Maternal posttraumatic stress response after the birth of a very low-birth-weight infant. J Psychosomatic Res. 2004, 57:473-476.

6. Wereszczak J, Shandor Miles M, Holditch-Davis D: Maternal recall of the neonatal intensive care unit. Neonatal Netw. 1997, 16:33-40.

7. Jubinville J, Newburn-Cook C, Hegadoren K, Lacaze-Masmonteil T:Symptoms of acute stress disorder in mothers of premature infants. Adv Neonatal Care. 2012, 12(4):246-53.

8. Charpak N, Ruiz JG, Zupan J, Cattaneo A, Figueroa Z, Tessier R, et. al. Kangaroo Mother Care: 25 years after. Acta Paediatr. 2005 May;94(5):514-22.

9. Norouzi F, Keshavarz M, SeyedFatemi N, Montazeri A. The impact of kangaroo care and music on maternal state anxiety. Complement Ther Med. 2013 Oct;21(5):468-72. doi: 10.1016/j.ctim.2013.07.006. Epub 2013 Aug 14.

10. de Alencar AE, Arraes LC, de Albuquerque EC, Alves JG. Effect of kangaroo mother care on postpartum depression. J Trop Pediatr. 2009 Feb;55(1):36-8. doi: 10.1093/tropej/fmn083. Epub 2008 Dec 9.

11. Lee SB, Shin HS. Effects of Kangaroo Care on anxiety, maternal role confidence, and maternal-infant attachment of mothers who delivered preterm infants. TaehanKanhoHakhoe Chi. 2007 Oct;37(6): 949-56. Korean. doi: 10.4040/jkan.2007.37.6.949. PMID: 17992067.

12. Johnson AN. The maternal experience of kangaroo holding. J ObstetGynecol Neonatal Nurs. 2007 Nov-Dec;36(6):568-73. doi: 10.1111/j.1552-6909.2007.00187.x. PMID: 17973700.

13. Roller CG.Getting to know you: mothers' experiences of kangaroo care.J ObstetGynecol Neonatal Nurs. 2005 Mar-Apr;34(2):210-7.

14. SN Vigod, L Villegas, C-L Dennis, LE Ross. Prevalence and risk factors for postpartum depression among women with preterm and low-birthweight infants: a systematic review. BJOG An International Journal of Obstetrics and Gynecology 2010.

15. De Guzman MLRE.A Validation of the Hospital Anxiety and Depression Scale (HADS) in the Medically-Ill. Acta Med Philipp. 2013; 47(3):53-62.

16. Smith-Nielsen J, Matthey S, Lange T, Vaever MS. Validation of the Edinburgh Postnatal Depression Scale against both DSM-5 and ICD10 diagnostic criteria for depression. BMC Psychiatry. 2018; 18:393.

17. Beck CT, Gable RK. Postpartum Depression Screening Scale: Development and Psychometric Testing. Nurs Res. 2000; 49(5):272-82. 